\title{
The Long CoN(S): A Case of Staphylococcus lugdunensis Endocarditis With Cerebral and Coronary Embolism
}

\author{
Jose Kuzhively $^{\mathrm{a}, \mathrm{c}}$, Sanjay A. Patel ${ }^{\mathrm{a}}$, Hrudya Abraham ${ }^{\mathrm{b}}$
}

\begin{abstract}
Staphylococcus lugdunensis is a coagulase-negative staphylococcus, which in humans can cause virulent infections frequently attributable to Staphylococcus aureus including superficial skin/soft tissue infections, infective endocarditis, osteomyelitis and endophthalmitis. A 61-year-old male with history of recent fixation of C1-C2 neck fracture, presented with sudden onset of right-sided weakness and fever. Examination revealed dense hemiplegia with UMN facial palsy of right side, and a pan-systolic murmur in the mitral area, radiating to the axilla. He had an elevated troponin-I of $18.8 \mathrm{ng} / \mathrm{mL}$ with right bundle branch block on EKG. Echocardiogram revealed mobile vegetation on the anterior leaflet of the mitral valve with ejection fraction of 55\% and no regional wall motion abnormality. He continued to have persistent high-grade fevers in spite of appropriate antibiotic therapy and blood cultures were persistently positive for $S$. lugdunensis for 5 days. He developed weakness of his left side after 2 weeks of hospital admission and MRA revealed left carotid dissection and complete occlusion of the right middle cerebral artery distal to the M1 segment which was embolic in nature. The frequency of S. lugdunensis infection is underappreciated and biofilm formation plays a major role in the pathogenesis. This is the first reported case of $S$. lugdunensis endocarditis complicated by NSTEMI from coronary embolism and simultaneous stroke from cerebral embolism. Due to the early, frequent and fatal complications from $S$. lugdunensis bacteremia, we might have to consider early surgical intervention in the absence of class I or IIa recommendation.
\end{abstract}

Keywords: Endocarditis; Staphylococcus lugdunensis; Vegetation; Stroke; Myocardial infarction

Manuscript accepted for publication August 26, 2014

aJohn H Stroger Hospital, Chicago, IL, USA

bMalankara Orthodox Syrian Church Medical College, Kolenchery, Kerala, India

${ }^{\mathrm{c} C o r r e s p o n d i n g ~ A u t h o r: ~ J o s e ~ K u z h i v e l y, ~ D e p a r t m e n t ~ o f ~ I n t e r n a l ~ M e d i c i n e, ~}$ John H Stroger Hospital, 1901 W Polk Street, Chicago, IL 60612, USA. Email: drjo999@gmail.com

doi: http://dx.doi.org/10.14740/jmc1903w

\section{Introduction}

Staphylococcus lugdunensis is a coagulase-negative staphylococcus (CoNS), which in humans ranges from a harmless skin commensal to a life threatening pathogen which was first described by Freney et al in 1988 on the basis of the analysis of 11 strains collected in Lyon, France .It can cause severe disease akin to the virulent infections frequently attributable to Staphylococcus aureus including osteomyelitis [1], prosthetic device infections [1], superficial skin/soft tissue infections [2], endocarditis $[1,2]$, intravascular catheter related bacteremia [3], central nervous system infections, peritonitis and endopthalmitis [4]. They also tend to colonize and cause infection below the waist [5]. S. lugdunensis is unique among CoNS because of its propensity for causing aggressive native valve infective endocarditis (IE). The frequency of $S$. lugdunensis infection is probably underappreciated, since many clinical laboratories do not routinely speciate CoNS $[6,7]$.

\section{Case Report}

A 61-year-old male with history of hyperparathyroidism, coronary artery disease, polysubstance abuse and recent fixation of C1-C2 neck fracture (3 months ago), presented with sudden onset of right upper and lower extremity weakness and fever. Examination revealed dense hemiplegia with UMN facial palsy of right side, and a pan-systolic murmur in the mitral area, radiating to the axilla. He had an elevated troponin-I of 18.8 $\mathrm{ng} / \mathrm{mL}$ with right atrial enlargement and right bundle branch block on EKG. Echocardiogram revealed large, spherical, mobile vegetation measuring $15.5 \times 11 \mathrm{~mm}$ on the anterior leaflet of the mitral valve (Fig. 1) with ejection fraction of $55 \%$ and no regional wall motion abnormality.

CT angiogram of the brain revealed dissection of the cervical, petrous, cavernous and supraclinoid portions of the left internal carotid artery with complete occlusion of the left middle cerebral artery (Fig. 2). He continued to have persistent high-grade fevers for 1 week in spite of appropriate antibiotic therapy and blood cultures were persistently positive for $S$. lugdunensis for 5 days. He developed weakness of his left side after 2 weeks of hospital admission and MRA revealed complete occlusion of the right middle cerebral artery distal to the M1 segment likely embolic in nature (Fig. 2). 


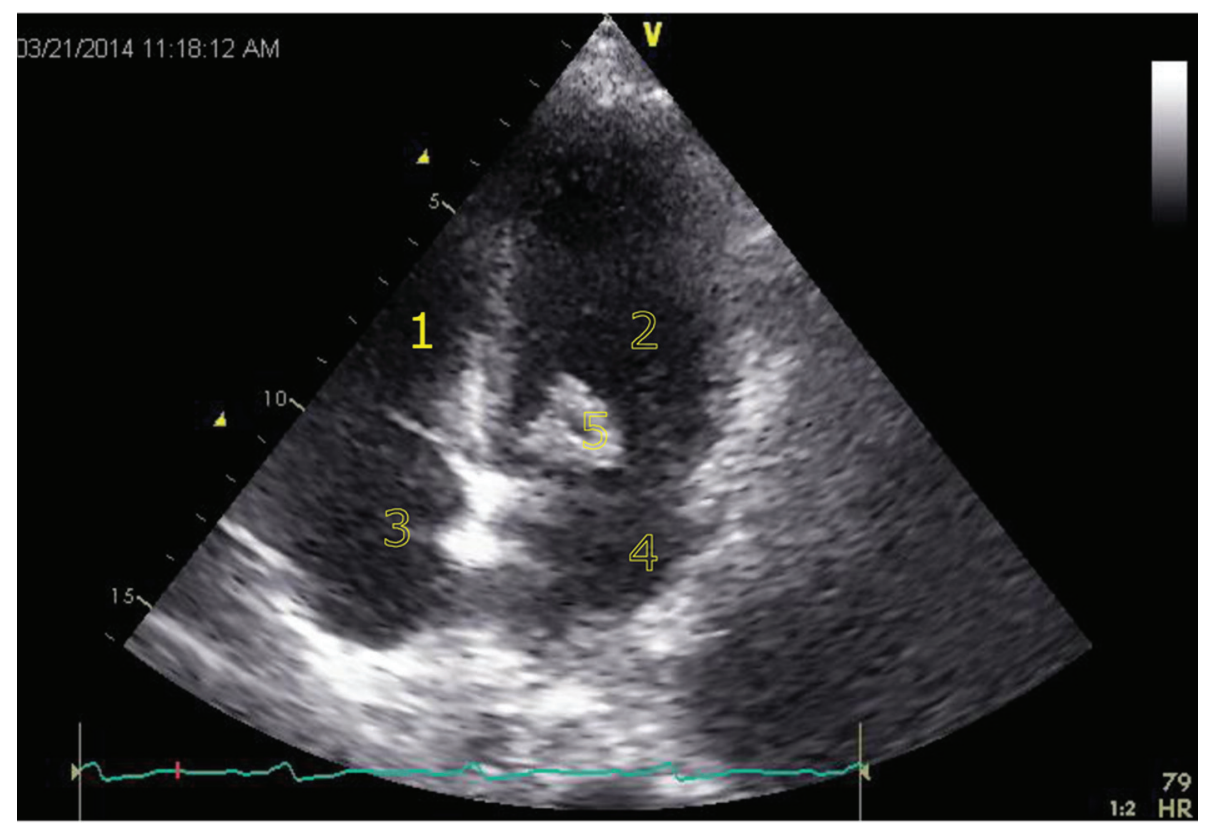

Figure 1. Transthoracic echocardiogram (apical four-chamber view) with mitral valve vegetation $(15.5 \times 11 \mathrm{~mm})$ over anterior mitral valve leaflet. 1. Right atrium. 2. Left atrium. 3. Right ventricle. 4. Left ventricle. 5. Mitral valve vegetation.

He was not considered a surgical candidate due to his comorbidities, bad prognosis and high intraoperative and perioperative mortality. Hence we treated him medically (oxacillin based on susceptibility for 6 weeks) for IE; however, his hospital course was complicated by aspiration pneumonia, ventilator support for respiratory distress, failed extubation and tracheostomy tube placement.

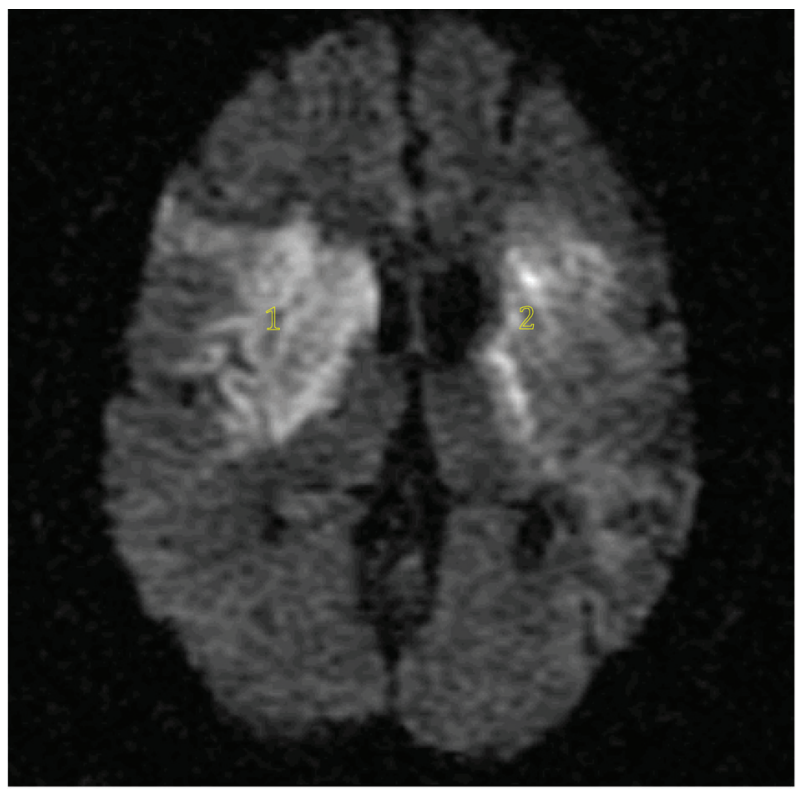

Figure 2. Diffusion-weighed image with bilateral acute infarcts in middle cerebral arterial territory. 1. Left middle cerebral arterial territory infarct for embolic stroke. 2. Right middle cerebral arterial territory infarct form left internal carotid artery dissection.

\section{Discussion}

S. lugdunensis is a Gram-positive, catalase-positive, coagulase-negative coccus. It is a non-motile, facultative anerobe that may demonstrate hemolysis on blood agar [8]. The frequency of $S$. lugdunensis infection is probably underappreciated, since many clinical laboratories do not routinely speciate CoNS $[6,7]$. The presence of clumping factor and variable performance characteristics of slide and rapid latex agglutination tests makes tube coagulation test important in distinguishing it from $S$. aureus [9-11]. Also phenotypic characteristics such as colony pleomorphism and $\beta$-hemolysis are useful when detecting $S$. lugdunensis, in contrast to clumping and synergistic hemolysis, which characterize several CoNS species.

Biofilm formation $[12,13]$ plays a major role in the pathogenesis of $S$. lugdunensis infection as it provides protection from antibiotics and host immunity and permits attachment of the organism on implantable devices. The biofilm of $S$. lugdunensis is composed of proteins rather than polysaccharide (which is a major component of biofilm produced by other organisms [13]).

When first described, S. lugdunensis was thought to be universally susceptible to penicillins and cephalosporins. But increased frequency of resistance to penicillin is being reported now. However resistance is increasing and some institutions report resistance to penicillin in about half of the isolates. It is important not only to identify $S$. lugdunensis in view of its clinical course, which is more aggressive than those of commonly isolated CoNS, but also to determine its susceptibility to oxacillin by detecting the mecA gene or its product, PBP2', since resistance has been reported [14]. Biofilm formation was increased in the presence of nafcillin, while it was decreased in the presence of tetracycline and linezolid. Moxifloxacin was 
the only antibiotic with activity against the S. lugdunensis organisms in biofilm; $73 \%$ of the biofilm isolates were susceptible [15].

Clinical infections due to $S$. lugdunensis include soft tissue infections, endocarditis, bacteremia, prosthetic device infections, central nervous system infections, peritonitis, endophtalmitis and osteomyelitis. S. lugdunensis endocarditis is an aggressive infection that affects native valves with greater frequency than prosthetic valves. It is found that prognosis of patients with IE and elevated troponins is very poor. Bacteremia without endocarditis (often related to an intravascular catheter) has a good prognosis.

Even though cases of $S$. lugdunensis endocarditis have been reported, no cases were reported in literature on $S$. lugdunensis endocarditis complicated by NSTEMI (trend in troponins with no evidence of myocarditis) and stroke from embolic phenomenon. Treatment of infection due to $S$. lugdunensis should be guided by the results of antibiotic susceptibility testing.

Surgical management for IE (class I) is indicated in Valve stenosis or regurgitation leading to heart failure, aortic of mitral regurgitation with elevation of left ventricular diastolic or atrial pressures, IE due to fungal or other resistant organisms, complications such as heart block, annular or aortic abscess, destructive penetrating lesions such as fistula from the sinus of Valsalva to the right or left atrium or right ventricle, mitral leaflet perforation with IE of the aortic valve, or infection in annulus fibrosis. Surgical management (class II b) can be considered for mobile vegetations larger than $10 \mathrm{~mm}$ with or without emboli [16].

\section{Conclusion}

S. lugdunensis is a skin commensal CoNS in humans that can become a life-threatening pathogen. It can cause severe disease similar to those attributable to $S$. aureus. The frequency of $S$. lugdunensis infection is probably underappreciated. This case demonstrates the importance of early identification and treatment of this virulent commensal. This is also the first reported case of $S$. lugdunensis endocarditis complicated by NSTEMI from coronary embolism and simultaneous cerebral embolism resulting in stroke. Even though surgical management in the absence of complication or persistent bacteremia is only class IIb recommendation based on American and European Heart Association guidelines, early surgical management should be considered in S. lugdunensis endocarditis, should no overwhelming class I indication exist, due to high rates of reported mortality.

\section{References}

1. Shah NB, Osmon DR, Fadel H, Patel R, Kohner PC, Steckelberg JM, Mabry T, et al. Laboratory and clinical characteristics of Staphylococcus lugdunensis prosthetic joint infections. J Clin Microbiol. 2010;48(5):1600-1603.
2. Bocher S, Tonning B, Skov RL, Prag J. Staphylococcus lugdunensis, a common cause of skin and soft tissue infections in the community. J Clin Microbiol. 2009;47(4):946-950.

3. Zinkernagel AS, Zinkernagel MS, Elzi MV, Genoni M, Gubler J, Zbinden R, Mueller NJ. Significance of Staphylococcus lugdunensis bacteremia: report of 28 cases and review of the literature. Infection. 2008;36(4):314-321.

4. Chiquet C, Pechinot A, Creuzot-Garcher C, Benito Y, Croize J, Boisset S, Romanet JP, et al. Acute postoperative endophthalmitis caused by Staphylococcus lugdunensis. J Clin Microbiol. 2007;45(6):1673-1678.

5. Bellamy R, Barkham T. Staphylococcus lugdunensis infection sites: predominance of abscesses in the pelvic girdle region. Clin Infect Dis. 2002;35(3):E32-34.

6. Gatermann SG, Koschinski T, Friedrich S. Distribution and expression of macrolide resistance genes in coagulase-negative staphylococci. Clin Microbiol Infect. 2007;13(8):777-781.

7. Kleiner E, Monk AB, Archer GL, Forbes BA. Clinical significance of Staphylococcus lugdunensis isolated from routine cultures. Clin Infect Dis. 2010;51(7):801-803.

8. Fleurette J, Bes M, Brun Y, Freney J, Forey F, Coulet M, Reverdy ME, et al. Clinical isolates of Staphylococcus lugdunensis and S. schleiferi: bacteriological characteristics and susceptibility to antimicrobial agents. Res Microbiol. 1989;140(2):107-118.

9. Mateo M, Maestre JR, Aguilar L, Cafini F, Puente P, Sanchez P, Alou L, et al. Genotypic versus phenotypic characterization, with respect to susceptibility and identification, of 17 clinical isolates of Staphylococcus lugdunensis. J Antimicrob Chemother. 2005;56(2):287-291.

10. Frank KL, Reichert EJ, Piper KE, Patel R. In vitro effects of antimicrobial agents on planktonic and biofilm forms of Staphylococcus lugdunensis clinical isolates. Antimicrob Agents Chemother. 2007;51(3):888-895.

11. Carroll K, Weinstein M. Manual and automated systems for detection and identification of microorganisms. In: Murray PR, Baron EJ, Jorgensen JH, et al, eds. Manual of clinical microbiology, 9th ed, Vol. 1. Washington, DC: ASM Press; 2009:192.

12. Gotz F. Staphylococcus and biofilms. Mol Microbiol. 2002;43(6):1367-1378.

13. Costerton JW, Stewart PS, Greenberg EP. Bacterial biofilms: a common cause of persistent infections. Science. 1999;284(5418):1318-1322.

14. Tee WS, Soh SY, Lin R, Loo LH. Staphylococcus lugdunensis carrying the mecA gene causes catheter-associated bloodstream infection in premature neonate. J Clin Microbiol. 2003;41(1):519-520.

15. Frank KL, Patel R. Poly-N-acetylglucosamine is not a major component of the extracellular matrix in biofilms formed by icaADBC-positive Staphylococcus lugdunensis isolates. Infect Immun. 2007;75(10):4728-4742.

16. Hoen B, Duval X. Clinical practice. Infective endocarditis. N Engl J Med. 2013;368(15):1425-1433. 